\title{
Synchronization in fiber laser arrays: theoretical study
}

\author{
Slaven Peleš ${ }^{a}$, Jeffrey L. Rogers ${ }^{b}$ and Kurt Wiesenfeld ${ }^{a}$ \\ ${ }^{a}$ School of Physics, Georgia Institute of Technology, Atlanta, GA \\ ${ }^{b}$ HRL Laboratories, LLC, Malibu, CA
}

\begin{abstract}
Fiber lasers have small size, high conversion rates and excellent thermal properties. On the other hand they generally produce smaller output intensity than semiconductor lasers. Recent experiments reported that a small number of fiber lasers can be synchronized simply by coupling them with an optical waveguide coupler near the output end. As a result the output peak intensity increases as a square of the number of coupled lasers, and well focused beams of high intensities can be produced, while preserving all other properties of fiber lasers. Synchronous behavior arises spontaneously, at constant pumping levels and without any active control. We investigate synchronization properties using a theoretical model, based on an iterated map with a particular symmetry. Our model captures key qualitative features seen in the experiments. We illustrate our results in a simple fiber laser configuration.
\end{abstract}

Keywords: laser arrays, coherence, synchronization, nonlinear dynamics, fiber lasers

\section{INTRODUCTION}

Producing large intensity laser beams has been a long standing goal of laser physics. One way to achieve this is to combine coherently several lasers, so that the peak output intensity increases as the square of the number of lasers. However, keeping different lasers inphase has proven difficult in practice. Various methods to control phases have been proposed, such as $\mathrm{MOPA}^{1}$ or injection locking. ${ }^{2}$

A significant milestone was achieved when it was demonstrated experimentally that a small number of fiber lasers, when suitably coupled, can synchronize spontaneously, without any active control. ${ }^{3-7}$ Limited success in spontaneous synchronization was reported earlier for semiconductor lasers. ${ }^{8}$

Although fiber laser synchronization can be reproduced utilizing a number different coupling schemes, the mechanism by which these lasers synchronize is not understood. What is common to all of the experiments is that the fiber lasers operate in a high gain regime, where nonlinear effects cannot be neglected. Earlier we introduced a dynamical model ${ }^{9}$ embodying the essential physics of this situation; mathematically, the resulting description is represented as a set of coupled nonlinear iterative maps.

We have performed a systematic investigation of the dynamics of coupled laser structures using this model. Three general types of behavior are observed: $(i)$ continuous wave output with constant gains, (ii) continuous wave output with variable gains and (iii) pulsing output. The synchronization properties of the laser array change significantly depending on which of these three types of behavior are involved. In this paper we describe the properties of these three types in a simple example of three coupled fiber lasers. Based on our results, we discuss possible strategies for coherent combining of fiber lasers and suggest experimental directions in this research area.

In Section 2 we describe our system and present the equation of motion. In Section 3 we study array behavior just above the lasing threshold, and derive analytical results for constant gain solutions. In Section 4 we investigate numerically more complicated dynamics that arise at high pumping levels. Finally in Section 5 we discuss the implications of our results for coherent combining of fiber lasers. 

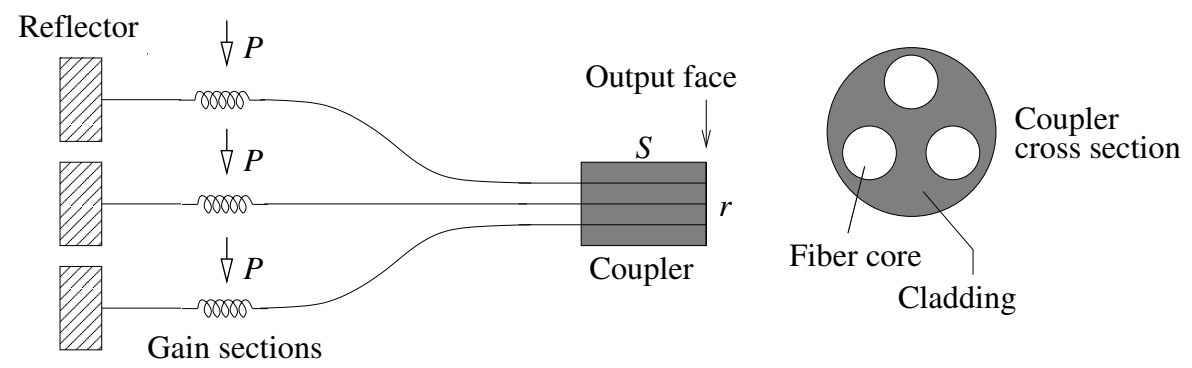

Figure 1. Fiber laser array. The coupler size is exaggerated for clarity.

\section{SYSTEM DESCRIPTION}

Motivated by recent experiments ${ }^{4,7}$ we consider the fiber laser array design depicted in Figure 1 . Three fiber lasers are coupled through a passive optical waveguide coupler near the output end, and terminated by a $100 \%$ reflective mirror at the other. The output face of the coupler is cleaved so that the field reflection coefficient is the same for all three fibers. Only about $20 \%$ of the fields are reflected back into the cavity, so the array operates in a high gain-high loss regime. The electromagnetic fields in the fibers mix only within the coupler, which makes up less than one per cent of the total cavity length.

The dynamics of this system can be described by an iterated map model ${ }^{9,10}$ :

$$
\begin{aligned}
E_{n}^{\prime} & =r e^{G_{n}} S_{n m} E_{m} \\
G_{n}^{\prime} & =G_{n}+\epsilon\left(P_{n}-G_{n}\right)-\frac{2 \epsilon}{I_{\mathrm{sat}}}\left(1-e^{-G_{n}}\right)\left|E_{n}\right|^{2}
\end{aligned}
$$

where $E_{n}$ is the complex field amplitude, $P_{n}$ is the pumping, and $G_{n}$ is the gain in the $n^{\text {th }}$ fiber. The prime sign denotes a map iteration, which corresponds to one roundtrip in the laser cavity. The parameter $\epsilon$ is the ratio between the roundtrip time inside the cavity and the fluorescence time for the lasing medium. Typical values for $\epsilon$ in the experiments under consideration are $\sim 10^{-4}$. $I_{\text {sat }}$ is the saturation parameter. We neglect losses in the individual fibers and in the coupler, so the field reflection coefficient $r$ represents the total loss in the cavity.

The elements of the coupling matrix $S_{n m}$ are in general complex numbers and need not have a direct physical interpretation. They can be obtained by integrating Maxwell equations over the entire length of the coupling region. For weak coupling the field equations inside the coupler can be written as ${ }^{11,12}$

$$
\frac{d E_{n}}{d t}-i \beta E_{n}=i \sum_{m} C_{n m} E_{m}
$$

where $\beta_{n}$ is a propagation constant for the $n^{\text {th }}$ fiber, and $C_{n m}$ is the coupling coefficient between $n^{\text {th }}$ and $m^{\text {th }}$ fibers. These coefficients can be estimated from the physical properties of the coupler. ${ }^{11}$ In what follows we assume that all three fibers are identical (so that $\beta_{n}=\beta$ ) and are evenly coupled $\left(C_{n m}=\kappa\right)$. If the coupling between fibers is uniform over the entire length of the coupler, the coupling matrix can be estimated as $S=e^{i 2 M d}$, where $d$ is the length of the coupler, $M_{n m}=\beta_{n} \delta_{n m}+\kappa$, and $\delta_{n m}$ is Kronecker's symbol. This yields matrix elements

$$
S_{n n}=\frac{1}{3} e^{i 2 d(\beta-\kappa)}\left(e^{i 6 d \kappa}-1\right) \quad \text { and } \quad S_{n m}=\frac{1}{3} e^{i 2 d(\beta-\kappa)}\left(e^{i 6 d \kappa}+2\right) \quad(n \neq m) .
$$

Before we turn to the array dynamics, it is instructive to look at the behavior of a single fiber laser, for which Eqs.(1-2) reduce to

$$
\begin{aligned}
E^{\prime} & =r e^{G} E \\
G^{\prime} & =G+\epsilon(P-G)-\frac{2 \epsilon}{I_{\text {sat }}}\left(1-e^{-G}\right)|E|^{2}
\end{aligned}
$$

Further author information: Slaven Peleš: E-mail: peles@cns.physics.gatech.edu, Telephone: 14043852508 

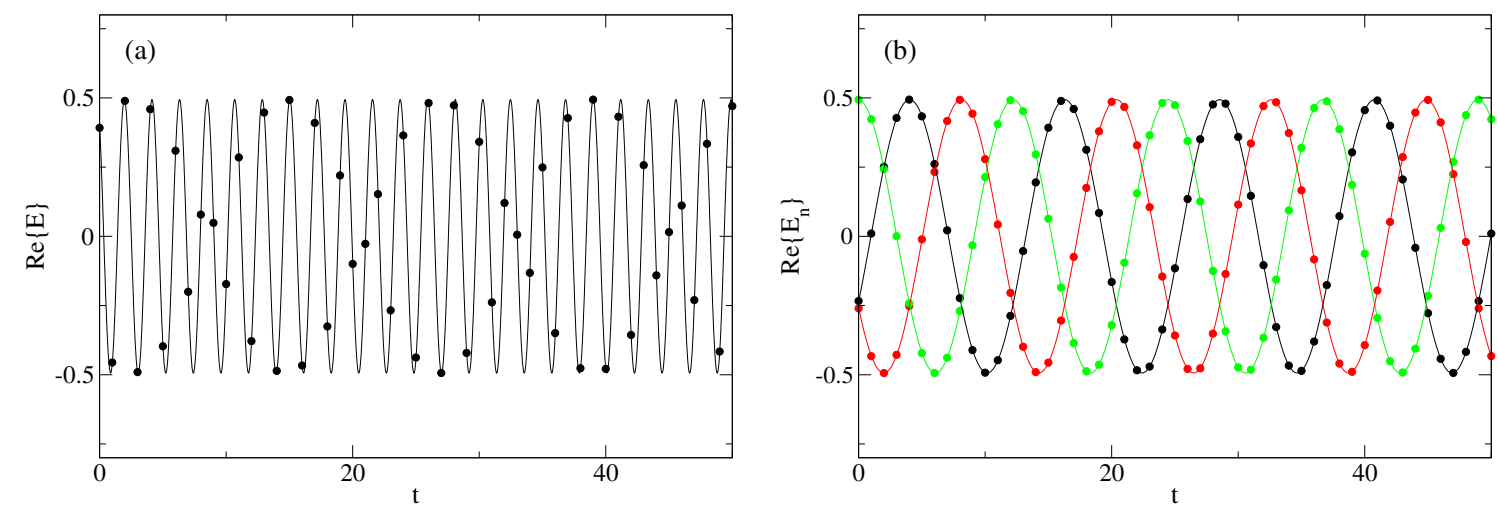

Figure 2. (a) Inphase and (b) splay phase solutions obtained for the same set of parameters, but different initial conditions. Both solutions are asymptotically stable and have a finite basin of attraction. Points represent map iterations, while curves are analytical solutions extended to continuous time. Pumping level is $P=2$, coupler length is $d=21510 \mu \mathrm{m}$, propagation constant is $\beta=8 \mu \mathrm{m}^{-1}$, and coupling constant $\kappa=0.001 \mu \mathrm{m}^{-1}$. The gain fields are constant in time and $G_{n}=\ln (1 / r)=1.609$.

This map has two fixed point solutions: the off-state solution $E=0, \quad G=P$, and the on-state solution

$$
G=\ln (1 / r) \text { and }|E|^{2}=\frac{P-\ln (1 / r)}{2(1-r)} I_{\text {sat }} .
$$

Note that the phase of the complex field amplitude of the on-state solution is arbitrary. The on state solution undergoes two transitions. ${ }^{9}$ The first one occurs at pumping $P_{1}=\ln (1 / r)$ and corresponds to the onset of lasing, while the second one happens for

$$
P_{2}=\ln (1 / r)+\frac{1-r}{2-3 r}
$$

and corresponds to the onset of pulsing. In our calculations we use $r=0.2$ (which is typical value for glass to air field reflection coefficient) so $P_{1}=1.609$ and $P_{2}=2.181$.

\section{SOLUTIONS NEAR THE LASING THRESHOLD}

In analogy with the fixed point solution to the single fiber system (7) we can find constant gain solutions to the array system (1-2) where $\tilde{G}_{n}=\ln (1 / r)$ for all fibers. Constant gain solutions are particularly interesting because they are analytically tractable, and they also give an insight into the nature of more complicated solutions. In the limiting case $\epsilon \rightarrow 0$ constant gain solutions become identical to eigenmode solutions in models where the gain dynamics is neglected. ${ }^{8}$ These solutions exist only for coupling configurations with a certain symmetry, because the complex field amplitudes $E_{n}$ have to satisfy eigenvalue equations for the coupling matrix $S$, and at the same time their magnitudes are fixed by (2). If all fibers are evenly pumped $\left(P_{n}=P\right)$, we can find constant gain solutions in the form

$$
\tilde{E}_{n}^{(q)}=R e^{-i \omega_{q} t+i 2 \pi n q / 3}, \quad n, q=0,1,2
$$

where $\omega_{0}=-2 d(\beta+2 \kappa), \omega_{1}=\omega_{2}=-2 d(\beta-\kappa)$, and $t$ is a discrete time expressed in terms of roundtrips. The index $n$ denotes the fiber; each index $q$ labels a solution to (1). Here $\tilde{E}_{n}^{(0)}$ is an inphase solution, while $\tilde{E}_{n}^{(1)}$ and $\tilde{E}_{n}^{(2)}$ are different splay phase* ${ }^{*}$ solution. These analytical solutions can be extended in a straightforward fashion to continuous time as shown in Figure 2.

For the purposes of laser synchronization we are most interested in determining the stability of the inphase solution $E_{n}^{(0)}$. Nonetheless, here we carry out calculations (at no extra cost) for all constant gain solutions. Our analytic results are valid for any number $N$ of all-to-all coupled fiber lasers; all of our numerical results are for the $N=3$ case. For simplicity in subsequent calculations we choose units in which $I_{\text {sat }}=1$. 

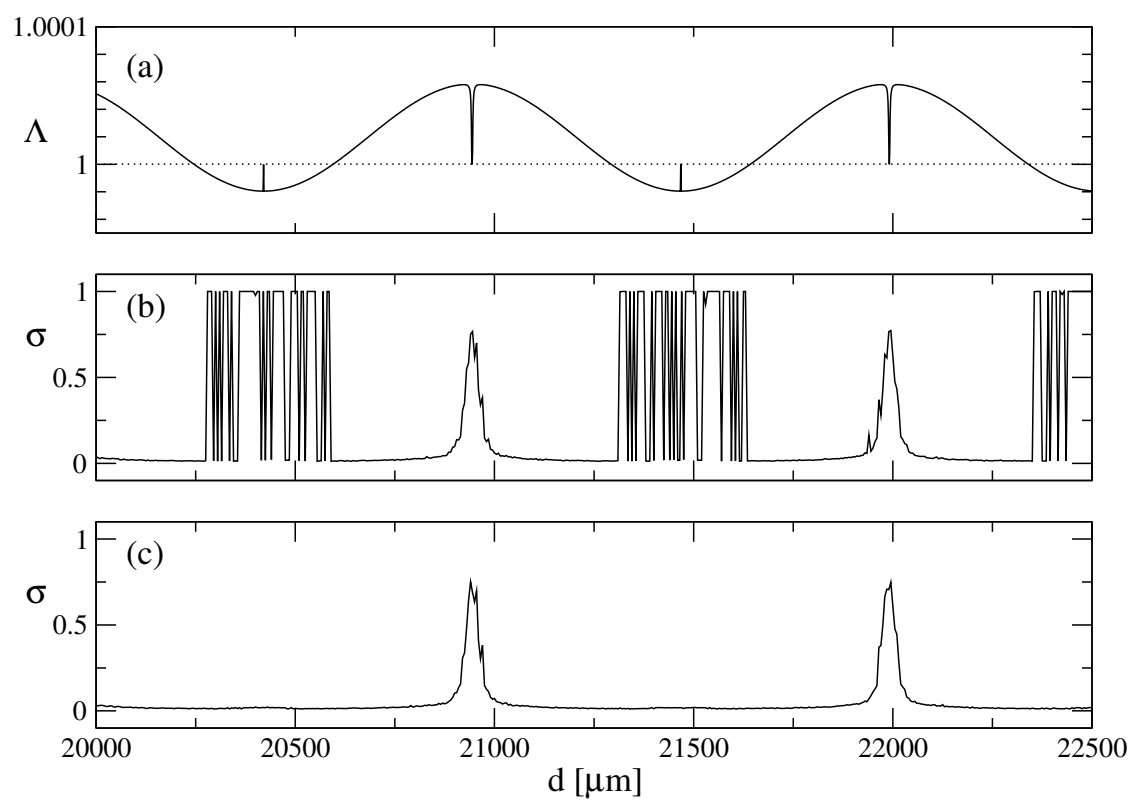

Figure 3. (a) Leading stability eigenvalue for the inphase solution plotted against coupler length $d$. Each fiber in the laser array is pumped at $P=2$. (b) Synchronization parameter plotted against coupler length for the pumping $P=10$ and initial conditions chosen near the inphase solution. (c) Synchronization parameter for $P=10$ and arbitrary initial conditions.

Small perturbations to the constant gain solutions $\eta_{n}=E_{n}-\tilde{E}_{n}^{(0)}, \gamma_{n}=G_{n}-\tilde{G}_{n}$ evolve according to the linearized equations

$$
\begin{aligned}
\eta_{n}^{\prime} & =r e^{\tilde{G}_{n}} \sum_{m} S_{n m} \eta_{m}+r e^{\tilde{G}_{n}} \gamma_{n} \sum_{m} S_{n m} \tilde{E}_{m}^{(q)} \\
\eta_{n}^{* \prime} & =r e^{\tilde{G}_{n}} \sum_{m} S_{n m}^{*} \eta_{m}^{*}+r e^{\tilde{G}_{n}} \gamma_{n} \sum_{m} S_{n m}^{*} \tilde{E}_{n}^{*(q)} \\
\gamma_{n}^{\prime} & =\gamma_{n}-\epsilon\left(1+2 e^{-\tilde{G}_{n}}\left|\tilde{E}_{n}\right|^{2}\right) \gamma_{n}-2 \epsilon\left(1-e^{-\tilde{G}_{n}}\right)\left(\tilde{E}_{n}^{(q)} \eta_{n}^{*}+\tilde{E}_{n}^{*(q)} \eta_{n}\right)
\end{aligned}
$$

This system of equations can be block diagonalized into $N 3 \times 3$ blocks by introducing new coordinates

$$
\psi_{n}^{(q)}=\frac{1}{\sqrt{N}} \sum_{k=0}^{N-1} \eta_{k} e^{i 2 \pi(n-q) k / N}, \quad \chi_{n}^{(q)}=\frac{1}{\sqrt{N}} \sum_{k=0}^{N-1} \eta_{k}^{*} e^{i 2 \pi(n+q) k / N}, \quad g_{n}=\frac{1}{\sqrt{N}} \sum_{k=0}^{N-1} \gamma_{k} e^{i 2 \pi n k / N}
$$

After switching to the new coordinates and substituting expressions for the $\tilde{G}_{n}$ and $\tilde{E}_{n}^{(q)}$ the system (10) becomes

$$
\begin{aligned}
\psi_{n}^{(q)^{\prime}} & =\lambda_{n-q} \psi_{n}+R e^{-i \omega_{q} t} \lambda_{q} g_{n} \\
\chi_{n}^{(q)^{\prime}} & =\lambda_{n+q}^{*} \chi_{n}+R e^{i \omega_{q} t} \lambda_{q}^{*} g_{n} \\
g_{n}^{\prime} & =\left[1-\epsilon\left(1+2 e^{-\tilde{G}_{n}}\left|\tilde{E}_{n}\right|^{2}\right)\right] g_{n}-2 \epsilon\left(1-e^{-\tilde{G}_{n}}\right) R e^{i \omega_{q} t} \psi_{n}^{(q)}-2 \epsilon\left(1-e^{-\tilde{G}_{n}}\right) R e^{-i \omega_{q} t} \chi_{n}^{(q)}
\end{aligned}
$$

where $\lambda_{q}=e^{-i \omega_{q}}$ is an eigenvalue of the coupling matrix $S$. We can remove the explicit time dependence by another coordinate transformation

$$
\psi_{n}^{(q)}=e^{-i \omega t} u_{n}^{(q)}, \quad \chi_{n}^{(q)}=e^{i \omega t} v_{n}^{(q)} .
$$

\footnotetext{
${ }^{*}$ Terms piston-phase and antiphase solutions are also common in the literature.
} 

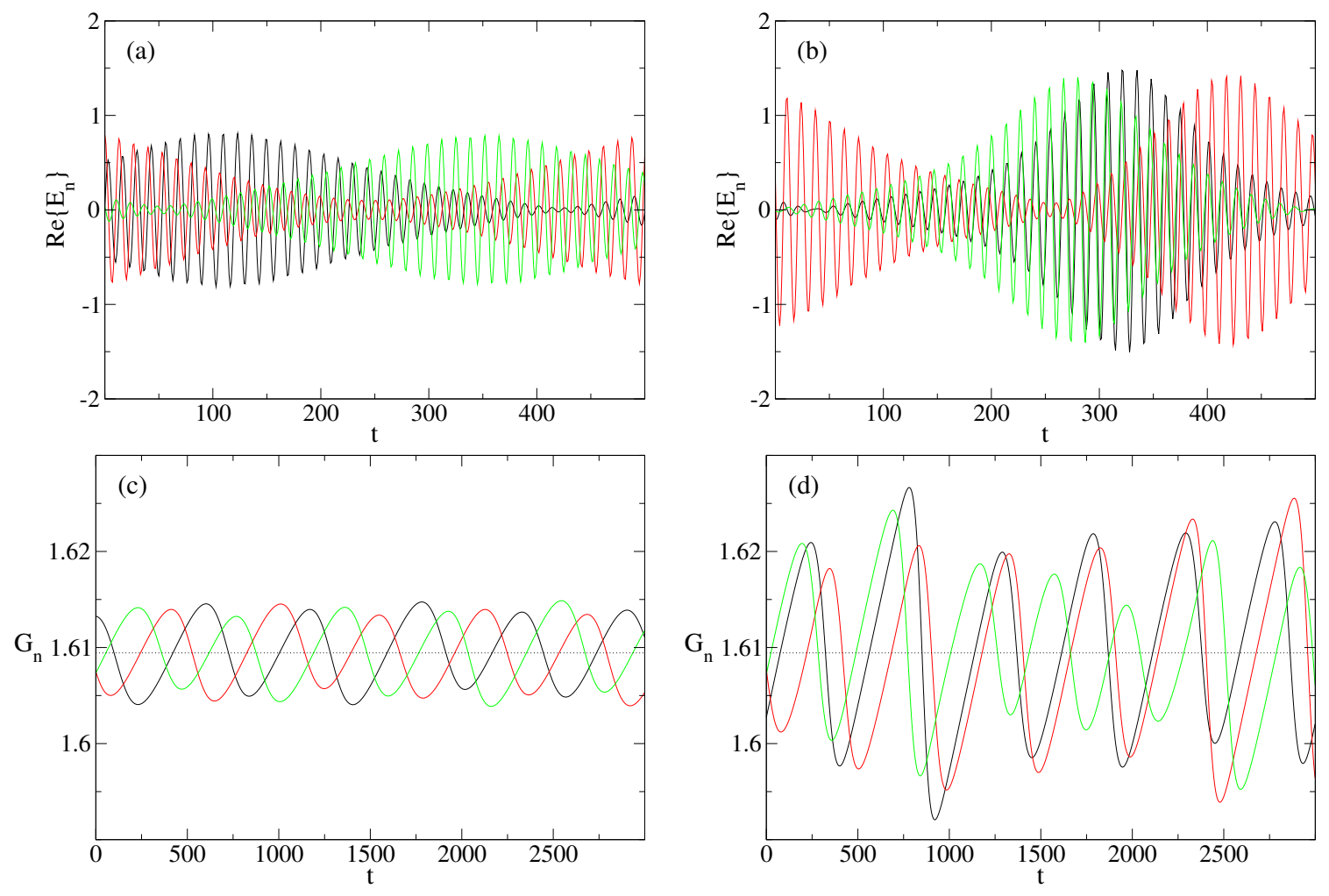

Figure 4. Time evolution of complex field amplitudes and gain fields for pumping $P=2$ (left panels), and $P=2.5$ (right panels). Horizontal lines in figures (c) and (d) describe lasing gain value $G_{n}=\ln (1 / r)$ for constant gain solutions. Continuous wave solutions with variable gain do not transition to pulsing solutions when the pumping is increased beyond

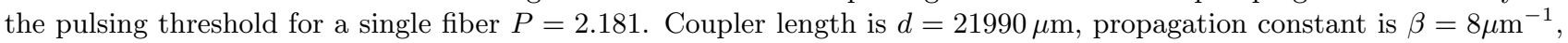
and coupling constant $\kappa=0.001 \mu \mathrm{m}^{-1}$.

so in the end the variational equations for the system (1-2) assume the simple form

$$
\begin{aligned}
u_{n}^{(q)^{\prime}} & =\lambda_{n-q} / \lambda_{q} u_{n}^{(q)}+R g_{n} \\
v_{n}^{(q)^{\prime}} & =\left(\lambda_{n+q} / \lambda_{q}\right)^{*} v_{n}^{(q)}+R g_{n} \\
g_{n}^{\prime} & =\left[1-\epsilon\left(1+2 r R^{2}\right] g_{n}-2 \epsilon(1-r) R u_{n}^{(q)}-2 \epsilon\left(1-e^{-\tilde{G}_{n}}\right) R v_{n}^{(q)}\right.
\end{aligned}
$$

where our notation implies taking modulo $N$ of $n-q$ and $n+q$. We can now easily read off blocks of the Jacobi matrix. For the case of the inphase solution $q=0$, the first block

$$
J_{0}=\left(\begin{array}{ccc}
1 & 0 & R \\
0 & 1 & R \\
-2 \epsilon(1-r) R & -2 \epsilon(1-r) R & 1-\epsilon\left(1+2 r R^{2}\right)
\end{array}\right),
$$

is exactly the same as the Jacobi matrix for the single fiber system. That means the inphase constant gain solution will be stable at most within the same pumping range $P_{1}<P<P_{2}$ as the on-state fixed point solution for a single fiber laser (5-6). The remaining blocks of the Jacobi matrix are

$$
J_{n>0}=\left(\begin{array}{ccc}
e^{-i 2 d N \kappa} & 0 & R \\
0 & e^{i 2 d N \kappa} & R \\
-2 \epsilon(1-r) R & -2 \epsilon(1-r) R & 1-\epsilon\left(1+2 r R^{2}\right)
\end{array}\right) .
$$

An obvious thing to note is that these blocks depend on the coupling parameters only through the periodic functions $e^{ \pm i 2 d N \kappa}$, so the stability eigenvalues obtained from these blocks have to be periodic functions of $d, N$ 

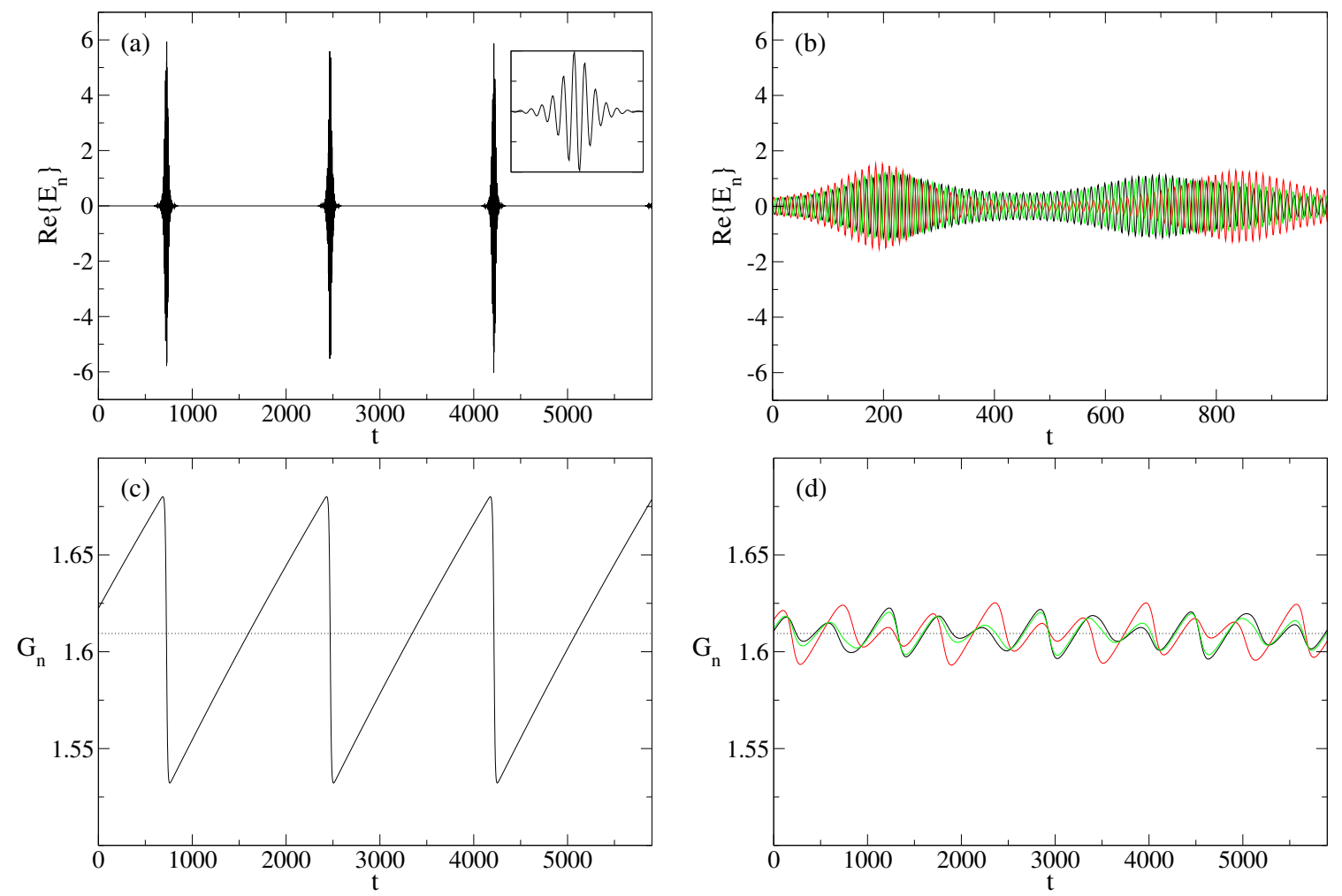

Figure 5. When the pulsing threshold for a single fiber laser is exceeded the constant gain inphase solution (Fig. 2) transitions into the pulsing inphase solution (left panels), while the splay phase solution transitions into the continuous wave solution with variable gain (right panels). The inset in (a) shows a typical pulse shape. Horizontal lines in figures (c) and (d) describe lasing gain value $G_{n}=\ln (1 / r)$ for constant gain solutions. Pumping level is $P=2.5$, while all the other parameters and initial conditions are the same as in Figure 2.

and $\kappa$ themselves. By calculating the eigenvalues for blocks $J_{n>0}$, we find that the inphase state is asymptotically stable when $d N \kappa \approx(m+1 / 2) \pi$, where $m=0,1,2, \ldots$, as shown in Figure 3a. It is interesting to note that the system is virtually uncoupled, and hence linearly marginally stable, whenever $d N \kappa=m \pi / 2$ exactly. This is observed in Figure 3a as spikes at maxima and minima of the leading stability eigenvalue.

By repeating this procedure for other constant gain solutions $(q=1,2)$, we find that all of them are asymptotically stable in the same parameter region as the inphase solution. This means that for those parameters multiple attractors coexist: the fields $E_{n}$ will exhibit inphase or splay phase oscillations depending on the initial conditions. In Figure 2b, for example, we obtained a splay phase solution by simply offsetting the gain fields by a small amount from the values used to produce Figure 2a, while keeping the other initial conditions the same.

Besides the constant gain solutions, in a pumping region near the lasing threshold there exists a number of other solutions with gain fields oscillating around the value $G=\ln (1 / r)$, such as those shown in Figure 4. Unlike the constant gain solutions, they generally cannot be represented in an analytical form, although some of those solutions can be well approximated by a linear combination of a few harmonics. Most often, these solutions have to be studied numerically. Our simulations suggest that variable gain solutions are stable in parameter regions opposite to those where the constant gain solutions are stable, that is for $d N \kappa \approx m \pi$ (Fig. 3). Another important difference is that variable gain solutions do not exhibit a transition at the pulsing threshold for a single fiber laser. Figure 4 shows that solutions below and above the pulsing threshold are qualitatively the same. 


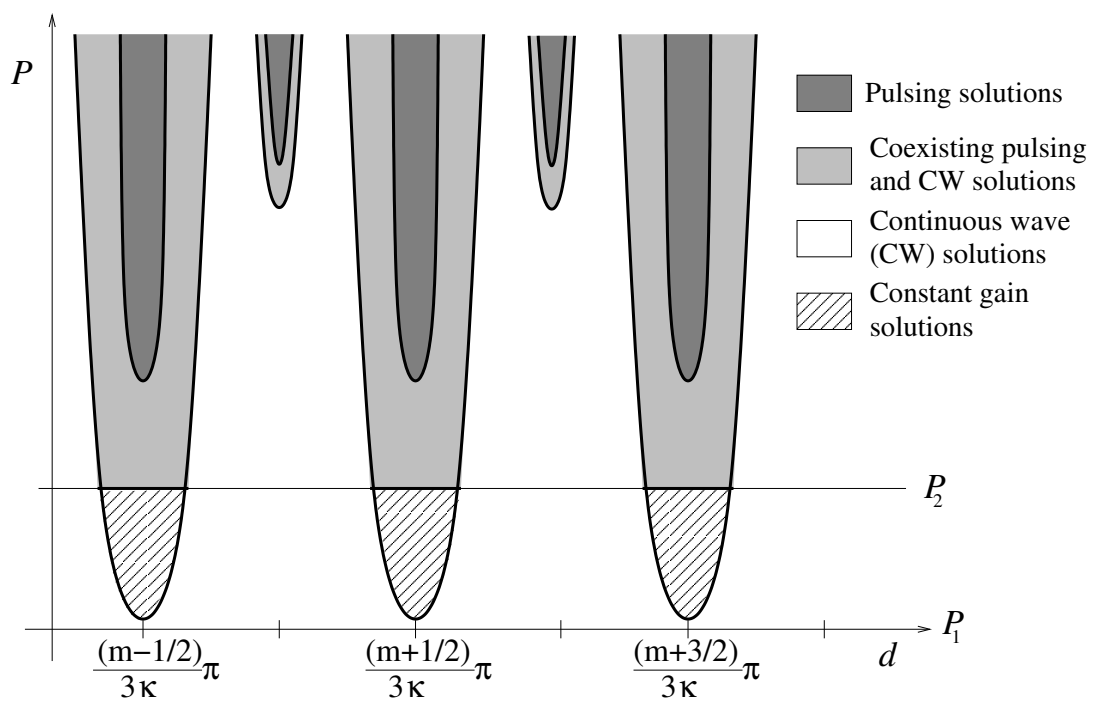

Figure 6. A sketch of stability regions in a parameter space for different types of solutions. As expected, continuous wave solutions are dominant at relatively low pumping values, while pulsing solutions are more likely to be found at high pumping levels. Stable constant gain solutions exist only in small regions just above the lasing threshold. The onset of pulsing for variable gain solutions depends on the coupling parameters. The pulsing threshold appears to be lower for more coherent solutions.

\section{LASER ARRAY DYNAMICS AT HIGH PUMPING VALUES}

The primary motivation for building laser arrays is to produce high power at the output. Therefore we turn our attention to the situation where the pumping level is several times the lasing threshold. Our analytical calculations show that constant gain solutions lose their stability already at pumping level $P_{2}=2.18$, which is less than twice the lasing threshold. The inphase constant gain solution transitions into an inphase pulsing solution such as the one shown in Figure 5a. This is not surprising: because of its symmetry the inphase solution should exhibit dynamics similar to a single fiber system. On the other hand, splay phase solutions transition to continuous wave solutions with variable gain (Fig. 5b). As the pumping level is increased further, splay phase solutions in a narrow band around $d=(m+1 / 2) \pi /(3 \kappa)$ transition into pulsing solutions. These transitions are summarized in Figure 6.

Since we do not have analytical expressions for the variable gain solutions, we cannot carry out a linear stability analysis analytically. Also, time series diagrams such as the one in Figure 4 give little information about the degree of phase coherence in the array. To study synchrony in such cases we introduce a synchronization parameter

$$
\sigma=\left\langle\frac{\left|\sum_{m} E_{m}\right|}{\sum_{m}\left|E_{m}\right|}\right\rangle_{t}
$$

where $\langle\cdot\rangle_{t}$ denotes the time average. The synchronization parameter takes on its maximum value one for inphase solutions, and becomes zero for splay phase solutions. We calculate $\sigma$ for different parameter values.

Our calculations suggest that the inphase pulsing solution is stable within the same parameter range as the constant gain inphase solution. In the example provided in Figure $3 \mathrm{~b}$ we calculate the synchronization parameter at a pumping level $P=10$ for a range of coupler lengths $d$. The initial conditions are chosen so that the phases of the complex field amplitudes $E_{n}$ are aligned to within 0.1 radians. The region on the diagram where the inphase solution appears to be stable is, however, riddled by near zero values for the synchronization parameter. This suggests that similar to the case of constant gain solutions, pulsing inphase solutions and splay-phase attractors coexist. We further conclude that the basin of attraction for the inphase solution is small and its boundaries change with the change of coupling parameters. If we repeat the same calculation for arbitrary initial conditions, typically we do not detect any inphase solutions, as shown in Figure 3c. 


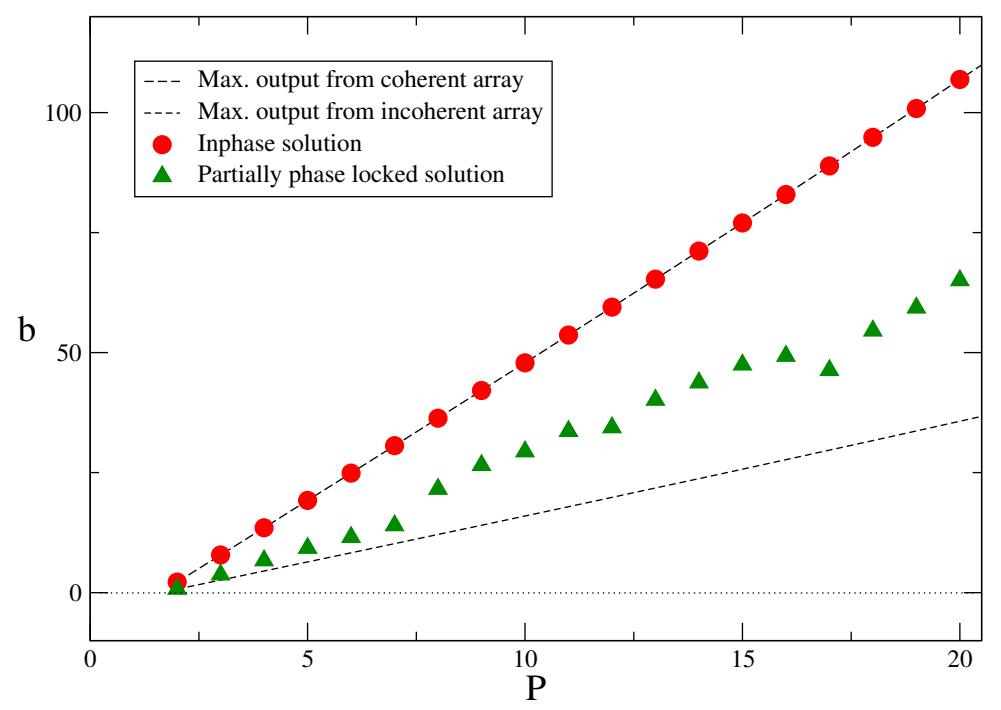

Figure 7. Output peak intensity vs. pumping parameter (arbitrary units). Coupler length is $21510 \mu \mathrm{m}$ for the inphase solution and $21990 \mu \mathrm{m}$ for the partially phase locked solution. The coupling coefficient is $\kappa=0.001 \mu \mathrm{m}^{-1}$.

Our numerical calculations also show that the system is robustly synchronized (albeit with a lesser degree of phase coherence) in the parameter region where inphase solutions are unstable. Similar to the previous case, we find there is a set of coexisting asymptotically stable solutions, only now all of these solutions have a similar degree of coherence. This means that, although different initial conditions result in the system settling down to a different attractor, the coherence of the system output remains virtually unchanged. These are continuous wave solutions with variable gains, such as the ones shown in Fig. 4. They transition to pulsing solutions at much higher pumping values than the constant gain solutions - over four times the lasing threshold (Fig. 6). The resulting pulsing solution has approximately the same degree of coherence.

Numerical simulations strongly suggest that the synchronization properties of our system do not depend on the pumping level, even in situations when continuous wave solutions transition to a pulsing solutions. This means that the synchronization conditions for the system can be obtained by studying system's dynamics just above the lasing threshold. This is particularly significant for inphase solutions, which are analytically tractable at low pumping values. To investigate this further we introduce a brightness parameter, ${ }^{9}$ defined as $b=\left\langle\left|\sum_{m} E_{m}\right|^{2}\right\rangle_{t}$. The brightness parameter is proportional to the peak intensity at broadside (zero angle). In Figure 7 we compare how array brightness changes as the pumping is increased for the two coherent solutions we found. The inphase solution produces maximum brightness; however, for typical initial conditions the system is most likely to be found in an antiphase solution with brightness having a near zero value. This corresponds to a defocused laser beam. For the robustly synchronous solution the brightness increases (approximately) linearly with the pumping, at a value in between the brightness of an inphase array and the brightness of an incoherent array.

\section{SUMMARY AND DISCUSSION}

We showed that a system of three coupled fiber lasers can exhibit two types of synchronization - pure inphase and a less coherent phase synchronization. Both solutions produced substantially improved peak output intensity compared to that of an incoherent array. As more fibers are added to the array, the output peak intensity scales as $N^{2}$ for the inphase solution and as $N^{\alpha}, 1<\alpha<2$ for partially phase locked solutions (Figure 8).

At low pumping values only constant gain solutions synchronize inphase, while all other coherent states are variable gain solutions. As the pumping is increased further, coherent states eventually transition into pulsing solutions with virtually the same degree of coherence. In general, we find that synchronization properties of the system do not change significantly with pumping parameter. 


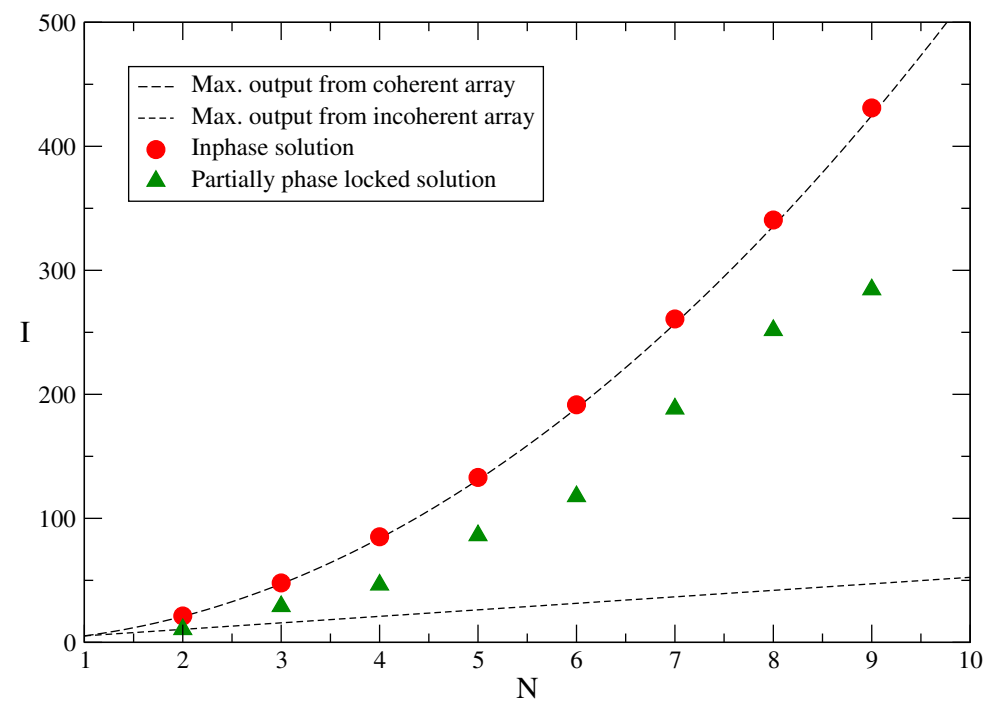

Figure 8. Output peak intensity vs. number of coupled fibers. The coupling is uniform all-to-all.

Fully symmetric inphase solutions have small basins of attraction and we conclude that they are unlikely to be detected in an actual experiment. The partially phase locked solutions lead to a more robust synchronization; however, they exist in narrower parameter regions.

An extensive numerical investigation we carried out indicates that these results are typical for more complex fiber laser structures. Summarizing results for various coupling configurations, however, is beyond the scope of this paper. Based on the present work, there are two strategies for coherent combining of fiber lasers that follow naturally. One is to produce arrays with globally stable inphase solutions. Theoretical progress in that direction has been already made for a hexagonal coupling configuration. ${ }^{10}$ The second approach is to design arrays which have partially phase locked solutions over a broad range of coupling parameters.

In practice qualitative changes in system's dynamics are easier to detect than quantitative changes. The results summarized in Figure 6 suggest that the onset of pulsing for the fiber laser array depends on coupling parameters, and there is a correlation between the pulsing treshold and the degree of coherence at the output. We believe that experimental investigation of critical system parameters at which these changes occur will provide valuable insight in synchronization mechanism for coupled fiber laser structure and help improve present models.

\section{ACKNOWLEDGMENTS}

SP would like to thank Amra Peles for critical reading of the manuscript and providing useful suggestions. SP also thanks Denis Tsygankov for a number of productive discussion.

\section{REFERENCES}

1. A. E. Siegman, Lasers, University Science Books, Mill Valley, CA, 1986.

2. Y. Liu, H. Liu, and Y. Braiman, "Injection locking of individual broad-area lasers in an integrated highpower diode array," Appl. Phys. Lett. 81, pp. 978-980, Aug. 2002.

3. H. Bruesselbach, D. C. Jones, M. S. Mangir, M. Minden, and J. L. Rogers, "Self-organized coherence in fiber laser arrays," Optics Lett. 30(11), pp. 1339-1341, 2005.

4. M. Minden, H. W. Bruesselbach, J. L. Rogers, M. S. Mangir, D. C. Jones, G. J. Dunning, D., L. Hammon, A. J. Solis, and L. Vaughan, "Self-organized coherence in fiber laser array," in Fiber Lasers: Technology, Systems, and Applications - Proceedings of SPIE, 5335, June 2004.

5. P. K. Cheo, A. Liu, and G. G. King, "A high-brightness laser beam from a phase-locked multicore yb-doped fiber laser array," Photon. Tech. Lett. 13, pp. 439-441, May 2001. 
6. E. J. Bochove, P. K. Cheo, and G. G. King, "Self-organization in a multicore fiber laser array," Optics Letters 28, pp. 1200-1202, July 2003.

7. V. A. Kozlov, J. Hernández-Cordero, and T. F. Morse, "All-fiber coherent beam combining of fiber lasers," Optics Lett. 24(24), pp. 1814-1816, 1999.

8. D. Mehuys, K. Mitsunaga, L. Eng, W. K. Marshal, and A. Yariv, "Supermode control in diffraction-coupled semiconductor laser arrays," Appl. Phys. Lett. , 1988.

9. J. L. Rogers, S. Peleš, and K. Wiesenfeld, "Model for high-gain fiber laser arrays," IEEE Journal of Quantum Electronics 41, pp. 767-773, June 2005.

10. S. Peleš, J. L. Rogers, and K. Wiesenfeld, "Robust synchronization in fiber laser arrays," Physical Review $E$, 2005. submitted.

11. A. W. Snyder and J. D. Love, Optical Waveguide Theory, Chapman and Hall, London, New York, 1983.

12. W.-P. Huang, "Coupled-mode theory for optical waveguides: an overview," J. Opt. Soc. Am. A 11, Mar. 1993. 